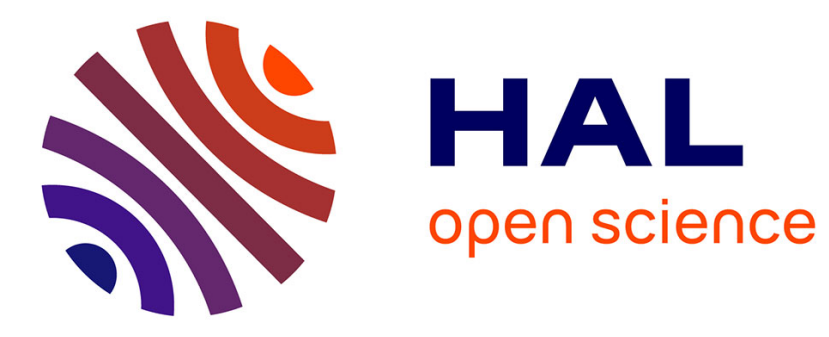

\title{
Liquid Resin Infusion process monitoring with superimposed Fibre Bragg Grating sensor
}

Emmanuel Marin, Laurent Robert, Sébastien Triollet, Youcef Ouerdane

\section{To cite this version:}

Emmanuel Marin, Laurent Robert, Sébastien Triollet, Youcef Ouerdane. Liquid Resin Infusion process monitoring with superimposed Fibre Bragg Grating sensor. Polymer Testing, 2012, 31 (8), pp.10451052. 10.1016/j.polymertesting.2012.07.018 . hal-00736788

\section{HAL Id: hal-00736788 \\ https://hal.science/hal-00736788}

Submitted on 29 Sep 2012

HAL is a multi-disciplinary open access archive for the deposit and dissemination of scientific research documents, whether they are published or not. The documents may come from teaching and research institutions in France or abroad, or from public or private research centers.
L'archive ouverte pluridisciplinaire HAL, est destinée au dépôt et à la diffusion de documents scientifiques de niveau recherche, publiés ou non, émanant des établissements d'enseignement et de recherche français ou étrangers, des laboratoires publics ou privés. 


\title{
Liquid Resin Infusion process monitoring with superimposed Fibre Bragg Grating sensor
}

\author{
Emmanuel MARIN ${ }^{\mathrm{a}}$, Laurent ROBERT ${ }^{\mathrm{b}, *}$, Sébastien TRIOLLET $^{\mathrm{a}, \mathrm{b}}$, Youcef OUERDANE $^{\mathrm{b}}$ \\ ${ }^{a}$ Université de Lyon, 42023 Saint-Etienne, France; UMR CNRS 5516 Laboratoire Hubert \\ Curien, Saint Etienne; Université Jean Monnet, Saint-Etienne. \\ ${ }^{b}$ Université de Toulouse; Mines Albi; ICA (Institut Clément Ader); \\ Campus Jarlard, F-81013 Albi cedex 09, France
}

\begin{abstract}
:
This paper reports on the instrumentation and monitoring of a fibreglass/epoxy composite manufacturing process using an optical fibre sensor based on a Bragg grating. The sensor is made of superimposed Long Period Grating (LPG) and Fibre Bragg Grating (FBG), both written on the same fibre zone. This sensor gives very accurate results and good spatial resolution, due to its ability to discriminate intrinsically between thermal and kinematic effects. The sensor was initially tested during the curing of an epoxy resin alone, in order to assess temperature and strain measurements. It is shown that these two factors can be well discriminated and quantified. The paper then presents results for monitoring Liquid Resin Infusion (LRI), with the FBG/LPG sensor embedded in a composite part. Dielectric analysis was also performed. Results and analyses of the thermal cycle of curing and induced residual strains are discussed.
\end{abstract}

Keywords: Polymer matrix composite; Optical fibre sensor; Smart material; Curing; Temperature strain discrimination; Residual stress

\footnotetext{
*Fax: +33 5634932 42, Email: laurent.robert@mines-albi.fr
} 


\section{INTRODUCTION}

Composite materials based on fibre glass/carbon with thermoset matrices are increasingly being used as structural materials, because of their light weight and high strength. However, there are some well known issues with these composites, such as how best to evaluate durability and damage tolerance design [1], how residual stresses are characterised [2] and how to identify the global mechanical properties of such composite structures [3]. These challenges are due to the multiplicity of the scales involved, the complexity of the mechanical answers, the unknown state of the material and the unavoidable property variability induced by the manufacturing processes that create both the material and the structure.

Among the various composite manufacturing processes, Liquid Resin Infusion (LRI) is a novel process having the major interest that components and sub-components are infused at the same time, giving better quality-controlled parts and reduced assembly steps. LRI is the moulding of high-performance composite parts by infusing liquid resin on dry (carbon or glass) preform instead of prepreg fabrics [4]. The composite manufacturing process remains complex through the reticulation phase, which brings mechanical strains exerted by tools and moulds, chemical strains (shrinkage) from resin contraction [5], and thermal strains exerted by the mismatch between the thermal expansion coefficients of the different components. The curing cycle then needs to be measured and controlled in order to obtain the final properties of the composite part, which are highly dependent on the process parameters, such as time duration, pressure and temperature. This requires in-situ instrumentation with sensors adapted for monitoring the composite manufacturing processes and assessing the thermal and mechanical factors. 
Optical fibre sensors are generally considered as alternative solutions to localize and measure the amplitudes of applied factors, particularly in applications where the information cannot be assessed directly. Because of their low intrusiveness, optical fibres can be easily embedded into the composite to perform in-situ and real-time measurements. Some optical sensors have already been used to monitor epoxy resin curing systems by the measurement of optical properties. These include sensors based on near-infrared spectroscopy (FTIR) [6], Raman spectroscopy [7], evanescent wave spectroscopy [8], or refractive index monitoring (Abbe- or Fresnel-based sensors, see [9] for example). Other optical sensors have been dedicated to monitoring temperature and strain. Among these, Bragg grating-based sensors can be considered as one of the most interesting candidates.

A standard Fibre Bragg Grating (FBG) is obtained by creating a submicron-period refractive-index modulation in the core of an optical fibre that couples the fundamental forward propagating core mode to the contra-propagating core mode. This coupling occurs at a specific wavelength $\lambda_{b}$, defined by the Bragg condition for the fibre grating, with the FBG acting as a narrow-band reflection filter. The Bragg wavelength is defined by the period of the FBG and the effective propagating-mode index, so that a change in either one of these parameters, induced for example by a change in temperature or strain, changes the Bragg wavelength. A Long Period Grating (LPG) has a higher period, typically a few hundred microns, which promotes coupling between the propagating core mode and co-propagating cladding modes. The attenuation of the cladding modes results in the transmission spectrum containing a series of bands, corresponding to the coupling to a different cladding mode, centred at discrete wavelengths. Similarly to the FBGs, these attenuation bands are sensitive to the local environment in terms of temperature and strain [10]. 
Although it is a great advantage that grating sensors are sensitive to several external factors, it is important to separate the thermal effect from the mechanical effect within the global information delivered by this kind of sensor. Several techniques have been described as a means to overcome this problem. These techniques can be separated into two main categories, according to whether the discrimination is intrinsic or extrinsic. The first consists of using two FBGs [11] or two LPGs [12]. These designs exploit different grating sensitivities to uncouple the strain/temperature effects. The FBG solution is known to exhibit low discrimination efficiency, while for the LPGs, although the solution presents a better discrimination efficiency, the sensor is more complicated to make as it uses two types of fibres [13]. Note that only the first category can ensure good spatial resolution, as the measurement can be performed in the same spatial zone. Other studies have been reported using both spatially-shifted LPG and FBG [14]. These designs only affect discrimination if the applied factors are non-homogenous, and lead to a comparatively poor spatial resolution of the measurement. As regards extrinsic discrimination techniques, these are generally performed by the use of two separate devices located in two separate places, leading to a nonoptimal spatial resolution of the measurement. The most commonly used all-fibre method consists of a pair of FBGs, one of which is encapsulated at the end of the fibre so that it remains free from mechanical stresses $[15,2,5]$. A thermocouple can also be linked to the FBG to collect the thermal component $[16,17]$. Another solution is to combine an FBG with another optical fibre sensor, for instance an Extrinsic Fabry-Perot Interferometer (EFPI) [18]. The main disadvantages of these techniques lie in their intrusive nature, in implementation difficulties, non-spatially localized measurements and difficulties in discriminating between different solicitations.

In this study, an experimental method is described for measuring strain and temperature applied to a composite part during manufacture by the LRI process. The method uses in-situ 
sensor instrumentation for extracting the thermal and mechanical characteristics of the composite material, mainly during the curing process, using a dual-period fibre Bragg grating. The originality of our technique lies in the use of two superimposed gratings (FBG and LPG) [13], allowing very good sensor spatial resolution with regard to the grating length, and high uncoupling efficiency.

In the following part, the principle of the sensor is detailed, along with the experimental configuration and calibration procedures. The experimental set-up required for curing the resin and for the LRI process is then described. Lastly, the results obtained with the embedded sensor during the curing of a pure epoxy resin, as part of the LRI process are presented and discussed.

\section{EXPERIMENTAL}

\subsection{Sensor Principle and calibration}

It is assumed that, in appropriate ranges (generally encountered in plastic and composites processes), the variation of the resonance wavelength $\lambda_{b}$ is linear with strain and temperature:

$$
\Delta \lambda_{b}=K_{\varepsilon} \Delta \varepsilon+K_{T} \Delta T
$$

where $K_{\varepsilon}$ and $K_{T}$ are the strain and temperature sensitivities, respectively, $\Delta \varepsilon$ and $\Delta T$ are the variations of the longitudinal strain and the temperature applied to the grating. It is supposed at this stage that the strain field around the sensor is isotropic and homogeneous, but this point will be the subject of discussion later in the paper. From Equation (1), it is clear that two equations are needed to calculate the unknown parameters. For this purpose, a sensor formed of two superimposed fibre gratings (a FBG and a LPG) was developed. The two resonance 
wavelengths shift with different variations of strain and temperature, and thus the relation between $\Delta \lambda_{b L}$ (for the LPG), $\Delta \lambda_{b F}$ (for the FBG), temperature and strain can be expressed as:

$$
\left[\begin{array}{l}
\Delta \lambda_{b L} \\
\Delta \lambda_{b F}
\end{array}\right]=\left[\begin{array}{ll}
K_{T L} & K_{\varepsilon L} \\
K_{T F} & K_{\varepsilon F}
\end{array}\right]\left[\begin{array}{l}
\Delta T \\
\Delta \varepsilon
\end{array}\right]=[K]\left[\begin{array}{l}
\Delta T \\
\Delta \varepsilon
\end{array}\right]
$$

where $K_{\varepsilon F}\left(K_{\varepsilon L}\right)$ and $K_{T F}\left(K_{T L}\right)$ are respectively the strain and the temperature sensitivities of the FBG (LPG). It is assumed that cross sensitivities can be neglected, as has been demonstrated experimentally [13]. It has been proven that the sensitivities of LPG and FBG are different for the same applied factor [19]. The solution to Equation (2) exists if the determinant $\mathrm{D}$ of matrix $[K]$ is not zero. Once the sensitivity coefficients are estimated by experimental calibrations, the applied variation of strain and temperature can be deduced.

All the sensors were made with the same experimental protocol as that described in [13]. Gratings were inscribed in an $\mathrm{H}_{2}$-loaded Corning ${ }^{\circledR}$ SMF-28 fibre with the help of the cw UV laser @244 nm. The LPG was first written with the point-by-point technique, and then the FBG was written over the entire length of the LPG using the well-known phase-mask technique with no fibre disassembly. Particular attention was paid to writing a relatively short LPG (12 mm) and an FBG of the same length, in order to obtain a small sensor with centimetre scale spatial resolution. Finally, the component was stabilized by heat treatment at $180^{\circ} \mathrm{C}$ for 3 hours to remove the unstable part of the grating. To determine the sensor sensitivities, a specific bench [13] was used to impose axial strain and/or temperature on the sensor. It was composed of a $1 \mu \mathrm{m}$ resolution-translation stage equipped with a micro-force sensor, using a heated glass capsule as a micro-oven. As an example, the sensitivity matrix of one of the superimposed LPG/FBG sensors that were developed is reported in Equation (3): 


$$
[K]=\left[\begin{array}{cc}
K_{T L}=39.3 \mathrm{pm} /{ }^{\circ} \mathrm{C} & K_{\varepsilon L}=-0.217 \mathrm{pm} / \mu \varepsilon \\
K_{T F}=9.75 \mathrm{pm} /{ }^{\circ} \mathrm{C} & K_{\varepsilon F}=1.18 \mathrm{pm} / \mu \varepsilon
\end{array}\right]
$$

It can be seen that the high decoupling efficiency of this sensor comes from the opposite strain sensitivities of both gratings, and from the fact that the LPG presents high thermal sensitivity for low strain sensitivity.

Sensor interrogation was carried out using a Leukos ${ }^{\circledR}$ compact supercontinuum whitelight source as a probe, with a bandwidth of $>700 \mathrm{~nm}$, and an optical-spectrum analyzer (Advantest $\left.^{(}\right)$Q8384) for detection purposes. Specific routines based on a Gaussian function were developed to fit transmission spectra. This adjustment procedure led to an estimation of $\lambda_{b}$ shift with a typical resolution better than $10 \mathrm{pm}$ for the LPG and $1.5 \mathrm{pm}$ for the FBG. The sensitivity errors were estimated to be $0.8 \mathrm{pm} /{ }^{\circ} \mathrm{C}$ and $0.3 \mathrm{pm} /{ }^{\circ} \mathrm{C}$ for temperature (LPG and FBG respectively), and $0.03 \mathrm{pm} / \mu \varepsilon$ and $0.01 \mathrm{pm} / \mu \varepsilon$ respectively for strain [20].

\subsection{Materials and Process}

The processed composite part was made of glass reinforcement with an epoxy resin. The resin was a HexFlow ${ }^{\circ}$ RTM6 from Hexcel ${ }^{\circledR}$ Composites, which is a premixed epoxy resin specifically dedicated to liquid-composite-moulding processes and commonly used in the manufacturing of composite materials, mainly in the aeronautics industry. The reinforcement was made with a non-crimp fabric glass fibre mat $[0 /-45 / 90 /+45]$ of $1 \mathrm{~mm}$ thickness. Two mats of $200 \times 200 \mathrm{~mm}$ square were symmetrically stacked to obtain a $2 \mathrm{~mm}$ quasi-isotropic composite plate part. In this case, the optical sensor was positioned in the symmetry plane of the glass preform. A thermocouple was also embedded near the optical sensor for comparison. Moreover, a flexible dielectric sensor was added in the vicinity of the optical sensor to assess 
the evolution of the curing process. In situ acquisition of the wavelength shifts, at a frequency of one spectrum per minute, was performed throughout the LRI process.

Two types of device were used in this work: one for testing only the curing of the resin and a second for the whole LRI process. For the curing tests, the resin was put in a silicon mould with two small holes and a sealant to place the optical LPG/FBG sensor, all of which was positioned in an oven. A micro-thermocouple with a $110 \mu \mathrm{m}$ wire diameter was also positioned in the vicinity of the optical sensor to validate and compare the results. Some studies (e.g. [17]) have reported the use of a single FBG and a thermocouple to discriminate temperature from strain during the composite manufacturing process and to determine residual strains. However, the device developed here seems to be an alternative way to avoid using an invasive (metal-based) thermocouple. In-situ acquisition was performed throughout the curing cycle.

The device used for the LRI process can be described as follows: an aluminium plate of 3 $\mathrm{mm}$ in thickness was used as a mould. It was treated with a release agent (Loctite ${ }^{\circledR}$ Frekote $^{\circledR}$ ) and the preform was placed on it. Sealant was added around the preform with a permeable draining fabric and a vacuum-bagging film placed on the top of the preform. Before infusion, the resin was preheated to $80^{\circ} \mathrm{C}$ in a heating chamber, connected to the vacuum bag inlet on one side, in order to decrease its viscosity and to make the infusion and the wetting of the glass fibre reinforcement easier. The liquid resin flow was then induced across the compressed preform thickness, also preheated to $80^{\circ} \mathrm{C}$, as a result of a prescribed vacuum pressure over the preform created by a vacuum pump connected to the vacuum bag outlet. After infusion of the preform by the resin, a second heating was applied up to $150^{\circ} \mathrm{C}$, followed by an isothermal curing phase of $120 \mathrm{~min}$ and, finally, by cooling to room temperature. 


\subsection{Dielectric sensor}

The dielectric sensor consists of two electrodes, with a sinusoidal voltage applied to one, while the resulting sinusoidal current is measured at the second. The response is attenuated in amplitude and shifted in phase according to the epoxy resin dielectric properties, due to the mobility of the ions and the alignment of the dipoles. These can be measured and the ionic viscosity can then be calculated (see [21] for details of the technique). In this work, a 10-

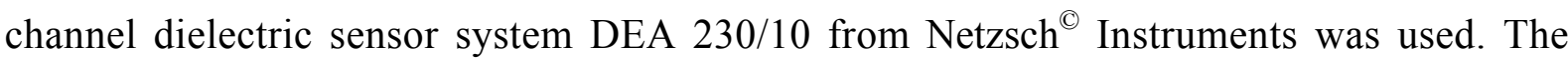
sensors chosen for the experiments were implantable, single-use sensors comprised of two interdigitated electrodes on a polyimide substrate (Idex). These sensors, of $10 \times 20 \mathrm{~mm}$ square, were simply placed between reinforcement plies. Dielectric measurements were conducted at various frequencies from $0.1 \mathrm{~Hz}$ to $10 \mathrm{kHz}$.

\section{RESULTS AND DISCUSSION}

This section is devoted to the presentation of the main results of the experiments: the monitoring of the resin curing alone, and the LRI-process monitoring, both using the opticalgrating-based sensor.

\subsection{Monitoring of simple epoxy resin curing}

In a first application, the LPG/FBG sensor was embedded in a Hexcel RTM6 resin for the purpose of monitoring the curing process. Figure 1 presents the temperature (measured by the thermocouple embedded near the optical sensor for comparison) and the FBG and LPG wavelength shift with time for the applied thermal cycle. The results shown in Figure 1 may be divided into three very distinct phases: the temperature increase (up to t1), the reticulation phase (cure plateau) from $t 1$ to $t 4$, and the temperature decrease. 
[Fig. 1 about here.]

In the first phase, when the temperature increases from $30^{\circ} \mathrm{C}$ to $130^{\circ} \mathrm{C}$ at around $2^{\circ} \mathrm{C} / \mathrm{min}$, the epoxy resin can be considered as liquid, so there is no detectable mechanical strain applied to the dual gratings $(\varepsilon=0)$. The measured shift of $\lambda_{b}$ only depends on temperature, which means that the shift of $\lambda_{b}$ may be written as Equation (4) with $K_{T}$ known from calibration:

$$
\Delta \lambda_{b}=K_{T} \Delta T
$$

The temperature increase phase can, therefore, be used as an in-situ temperature calibration of the sensor in its environment.

During the second phase (the cure plateau generally associated with the reticulation phase), two distinct thermal states can be observed. The first is an overshoot of the temperature from $\mathrm{t} 1$ to $\mathrm{t} 3$, which corresponds to the exothermal manifestation of chemical bonding, while the second state can be considered as isothermal at $130^{\circ} \mathrm{C}$ from $\mathrm{t} 3$ to $\mathrm{t} 4$. However, in most cases studied, the overshoot does not appear to be as high as it was in this experiment. This difference can be explained, firstly by our use in this case of only pure resin, without reinforcement (a material with low thermal conductivity), and secondly by the facilitation of temperature diffusion caused by the large heat flux exchanged between the sample and the metallic mould, a phenomenon that cannot occur in a silicon mould. However, it is worth pointing out that, contrary to metallic moulds, this kind of silicon mould allows free deformations during the whole curing cycle. Obviously, in our experiment, the resin reticulation did not occur at a constant temperature. During the reticulation phase, the epoxy 
resin continuously changes from its liquid state to its reticulated state, which simultaneously fixes on the optical fibre and allows strain transfer [2], leading to a continuous change in the behaviour of the material surrounding the sensor. The total strain applied to the sensor is the sum of a mechanical part (resin chemical shrinkage) and a thermal part (increase and decrease in the temperature) in a material whose stiffness is time-dependent. This is highlighted by the negative wavelength shift for the FBG from $t=t 2$, along with the positive wavelength shift for the LPG (because the strain sensitivity of the LPG is negative), as depicted in Figure 1.

During the isothermal phase ( $\Delta T=0$ ), the wavelength shift is expressed by Equation (5), which depends only on the mechanical strain:

$$
\Delta \lambda_{b}=K_{\varepsilon} \Delta \varepsilon
$$

The curing ends after the plateau $\left(t>t_{4}\right)$, with the decrease in temperature (third and last phase), where the cure residual stresses are proportional to the measured residual strains [2]. In this case, the wavelength shift depends on strain and temperature. Under the hypothesis of a surrounding material volume considerably greater than the optical fibre volume (in such a case the effective behaviour of the assembly is essentially governed by the surrounding media [22]), it can be expressed as:

$$
\begin{aligned}
\Delta \lambda_{b} & =K_{T} \Delta T+K_{\varepsilon} \Delta \varepsilon+K_{\varepsilon}\left(\alpha_{\text {media }}-\alpha_{\mathrm{SiO}_{2}}\right) \Delta T \\
& =K_{\varepsilon} \Delta \varepsilon+K_{T}^{*} \Delta T
\end{aligned}
$$

The additional thermal-strain effect generally induces an increase in the temperature sensitivity, leading to the apparent temperature sensitivity, noted $K_{T}^{*}$, linked to the surrounding media. 
From the curves of the FBG and LPG wavelength shifts, presented in Figure 1, and knowing the two temperature and the two strain sensitivities from calibration, as presented in Equation (3), discrimination can then be achieved. Figure 2(a) shows the temperature response of our sensor (quoted FBG\&LPG in Figure 2a) during the curing cycle, while the total strain is reported in Figure 2(b).

[Fig. 2 about here.]

The results presented in Figure 2(a) are validated by comparison with the thermocouple response for temperature (quoted TC in Figure 2(a)). The results for the strain measurement presented in Figure 2(b) are also validated by comparing the strain measured with our optical sensor and the strain deduced from the single FBG wavelength shift using the thermocouple to measure the temperature and to discriminate both factors. It is worth pointing out that these results are quite good, except for the temperature response during the overshoot, where the optical sensor and thermocouple responses differ slightly. This may be explained by the fact that the two sensors are not positioned strictly at the same location. These results and analyses suggest the following comments.

- The measured strain is not affected by the temperature variation up to $t=t 2$, which corresponds to the maximum temperature of the exothermic reaction.

- Between $\mathrm{t} 2$ and $\mathrm{t} 3$ we observed a contraction of about $3600 \mu \varepsilon$ with a decrease in temperature of about $20^{\circ} \mathrm{C}$. This is certainly the result of both the chemical contraction coming from the thermoset reticulation and the thermal contraction induced by the surrounding material along with the decrease in temperature.

- Between $\mathrm{t} 3$ and $\mathrm{t} 4$ both strain and temperature are constant, which is consistent with the fact that there is no wavelength shift of both gratings, as seen in Figure 1. 
- The temperature decrease and the strain from a thermal origin occur from $t 4$, following Equation (6). This thermal strain has an amplitude of about $6500 \mu \varepsilon$, giving more than $1 \%$ for total strains back to ambient temperature. These large values can be explained by the fact that strains are free to develop in the silicon mould, and, of course, because there are no reinforcement fibres with generally lower thermal expansion and higher stiffness in comparison to the matrix.

This analysis can be extended by plotting the measured total strain with the temperature, as presented in Figure 3.

[Fig. 3 about here.]

Inserted in the figure are the slopes calculated during the exothermic part of the isothermal phase and during the cooling phase. During the latter phase, the epoxy resin is solid and its coefficient of thermal expansion can be estimated at $\alpha_{\text {resin }}=63 \mu \varepsilon /{ }^{\circ} \mathrm{C}$ which is in agreement with values from the literature [23]. However it is shown here that the resin is not totally cured at $\mathrm{t}=\mathrm{t} 2$, nor between $\mathrm{t} 2$ and $\mathrm{t} 3$, because the decrease in temperature after the exothermic overshoot induces an apparent (much higher) coefficient of thermal expansion of $180 \mu \varepsilon /{ }^{\circ} \mathrm{C}$ (see Figure 3). This represents the contribution of the chemical contraction due to conversion, added naturally to the thermal contraction. Between $\mathrm{t} 2$ and $\mathrm{t} 3$ the measured strain is equal to $3600 \mu \varepsilon$ for a temperature drop of $20^{\circ} \mathrm{C}$, which would thus be at the origin of a thermal contraction of $20 \times \alpha_{\text {resin }}=1260 \mu \varepsilon$, assuming a constant coefficient of thermal expansion from $\mathrm{t} 2$ to the end of the curing. One may conclude that, between $\mathrm{t} 2$ and $\mathrm{t} 3$, about $1300 \mu \varepsilon$ are provided by the thermal decrease and about $2300 \mu \varepsilon$ by the chemical reaction itself. 


\subsection{Liquid Resin Infusion monitoring}

As good results were obtained from sensor tests during the epoxy curing, the FBG/LPG sensor could now be embedded in a glass/epoxy (RTM6)-composite part made using the process of Liquid Resin Infusion (LRI), as previously described. Figure 4 reports the temperature measured by the thermocouple, along with the FBG and LPG wavelength shifts as a function of time during the whole process.

[Fig. 4 about here.]

The DEA analysis, along with the temperature evolution measured by the thermocouple, is reported in Figure 5.

[Fig. 5 about here.]

First, the ionic viscosity presents a large decrease just after the beginning of the heating cycle. The resin viscosity reaches a minimum at $\mathrm{t}_{1}=30 \mathrm{~min}$, which corresponds to the beginning of the isothermal curing. During the reticulation phase, the viscosity reaches its maximum level, leading to the end of the curing: the resin is now completely solid. Figure 5 highlights an inflexion point during the reticulation phase near $t_{2}=60 \mathrm{~min}$, which corresponds to the gelation time [21] (the change point from the liquid phase to the rubber phase). At this time, the velocity of the cross-linking reaction is at its highest, corresponding to a maximum variation in the fractional conversion with time. This also involves a maximum heat flux induced by the reaction [24], as can be seen from the maximum temperature reached at $t_{2}=60$ min in Figure 5. Finally, Figure 5 exhibits no variation from $t_{4}=130 \mathrm{~min}$, corresponding to the final solid state of the resin. 
From the raw wavelength-shift results shown in Figure 4, and with the sensitivity matrix of Equation (7)

$$
[K]=\left[\begin{array}{cc}
K_{T L}=50.9 \mathrm{pm} /{ }^{\circ} \mathrm{C} & K_{\varepsilon L}=-0.266 \mathrm{pm} / \mu \varepsilon \\
K_{T F}=11 \mathrm{pm} /{ }^{\circ} \mathrm{C} & K_{\varepsilon F}=1.21 \mathrm{pm} / \mu \varepsilon
\end{array}\right]
$$

obtained after the sensor calibration, discrimination of temperature and strain can then be achieved.

Figure 6a shows the temperature response of our sensor (quoted LPG\&FBG in Figure 6a) during the whole curing process, while the total strain is reported in Figure 6b.

[Fig. 6 about here.]

The results presented in Figure 6a are validated by comparing the thermocouple temperature response (quoted TC in Figure 6a). Results for the strain measurement presented in Figure $6 \mathrm{~b}$ are also validated, by comparing the strain measured with our optical sensor (quoted LPG\&FBG in Figure 6b) and the strain deduced from the single FBG wavelength shift, using the thermocouple to measure the temperature and to discriminate between each factor (quoted TC\&FBG in Figure 6b). The enlargement of Figure 6a and 6b (presented in Figure $6 \mathrm{c}$ and $6 \mathrm{~d}$ ) highlights a decrease of about $100 \mu \varepsilon$ in the measured strain during the isothermal plateau $(\Delta T=0)$. These results show that the measurable contraction starts at $t_{2}=$ $60 \mathrm{~min}$ and stops at $\mathrm{t}_{4}=130 \mathrm{~min}$, demonstrating that the measured strains are coming from the chemical contraction of the epoxy resin. One may deduce that these two process periods correspond, respectively, to the gelation time and to the end of the conversion of the resin, which is confirmed by the dielectric analysis results presented in Figure 5. Note also that the chemical contraction estimated by the optical fibre sensor is very limited, mainly because the 
reinforcement fibres present a higher stiffness in comparison to the matrix, leading to the matrix being in a state of tension at the end of the conversion.

After cooling, results for residual strains, which lead to cure residual stresses, can be calculated at $2250 \mu \varepsilon$ (see Figure 6b). This value is lower than that reported for the pure epoxy resin curing because the coefficient of thermal expansion of the glass fibre is lower than that of the resin. In the case of a composite part provided by the LRI process, the measured strain coming from the chemical contraction of the epoxy resin is very weak, because it is prevented by glass reinforcement fibres. Thus, its detection remains difficult, contrarily to the case of curing pure epoxy resin. It is confirmed that the major share of residual stress in the reinforcement fibres is attributed to variations taking place at the time of the cooling-down phase, due to the mismatch between coefficients of thermal expansion and mould / composite interactions.

However, it is noticeable that the matrix remains submitted to high positive stress, which originates from both the chemical contraction and the thermal contraction. The former is about $2300 \mu \varepsilon$ (estimated in section 3.1) during the pure epoxy resin curing, while in the case of the composite it is almost totally bound by reinforcement. The latter induces about $6500 \mu \varepsilon$ during the cooling phase of the pure epoxy resin (see again section 3.1) and $2250 \mu \varepsilon$ in the case of the composite, leading to a tensile strain of about $6500-2250=4250 \mu \varepsilon$ subjected to the resin.

Finally, it should be emphasised that, as mentioned before, there may be both straininduced polarization (e.g. transverse strain), as the composite part is anisotropic [25], and also non-homogeneous strain along the fibre sensor [26], as the sensor presents a relatively long length $(12 \mathrm{~mm})$ compared to the dimension of the composite part. To investigate these 
possibilities, the evolution of the Bragg spectra shapes recorded during the LRI process was assessed. Note that it was the spectrum shape itself that was analyzed and not the power level of the reflected optical signal, as the former may reveal a complex strain state around the optical sensor. Normalised spectra of both gratings during the curing are presented over several times in Figure 7.

[Fig. 7 about here.]

Normalisation of the wavelengths was achieved by keeping the wavelength range constant for all the spectra, allowing changes in shape to be more clearly observed: $80 \mathrm{~nm}$ for the LPG spectra and $12 \mathrm{~nm}$ for the FBG spectra. A precise observation of Figure 7(a) shows that there is a small change in the LPG spectra: there is no significant variation between times 0, 100 and $200 \mathrm{~min}$ (heating and isothermal phases) nor between times 300 and $400 \mathrm{~min}$ (cooling phase). However, a small enlargement is observed between times 0, 300 and 900: a $20 \%$ and $35 \%$ increase in the full width at half maximum (FWHM). The exact same tendencies are observed in the FBG spectra in Figure 7(b). This is certainly the signature of transverse strains which remain small in comparison to the longitudinal strains that occur during the cooling to room temperature. The spectra shapes are globally conserved, indicating that transverse effects due to contraction during cooling have no significant influence on the longitudinal strain measurement. Our strain measurements can thus be considered as trustworthy.

\section{CONCLUSIONS}

This paper describes the monitoring of a LRI composite manufacturing process. It focuses on the instrumentation of this process, which is carried out using an optical fibre 
sensor based on dual association of long and short period gratings. These were used to discriminate the temperature from the strain during the process. Our sensor was calibrated to obtain sensitivity responses before embedding, first in epoxy resin, then in a fibreglass/epoxycomposite part for LRI process monitoring.

The discrimination of the sensor temperature and strain responses was validated during a simple epoxy resin curing by comparing them with thermocouple measurements. The sensor presents an accurate monitoring response and low intrusiveness, while dispensing with the use of a thermocouple. Analysis of the curing allowed strains from chemical origins (shrinkage) and thermal origins to be assessed. The LRI process was also monitored with the superimposed grating sensor and with a dielectric sensor for comparison. The developed sensor presents a good and accurate response for the monitoring of such a process. It was also shown that the grating-based sensor highlights the two significant points of the process, which are the gelation time and the end of solidification. Finally, a wavelength-spectra analysis was carried out and showed that transverse effects (due to contraction during the cooling) have no significant influence on the longitudinal strain measurement, thus guaranteeing a high degree of confidence in the strain measurements.

\section{Acknowledgment:}

The authors wish to acknowledge the French Ministry of the Economy, Finance and Industry, and the French Midi-Pyrenees Region for their financial supports.

\section{References}


[1] S. Takeda, Y. Okabe, N. Takeda, Delamination detection in CFRP laminates with embedded small-diameter fiber Bragg grating sensors, Comp. Part A: Appl. Sci. Manuf. 33 (2002) 971.

[2] M. Mulle, F. Collombet, P. Olivier, Y.-H. Grunevald, Assessment of cure-residual strains through the thickness of carbon-epoxy laminates using FBGs Part I: Elementary specimen, Comp. Part A: Appl. Sci. Manuf. 40 (2009) 94.

[3] M. Mulle, R. Zitoune, F. Collombet, L. Robert, Y.-H. Grunevald, Embedded FBGs and 3D DIC for the stress analysis of a structural specimen sub jected to bending, Comp. Struct. 91 (2009) 48.

[4] C. Williams, J. Summerscales, S. Grove, Resin Infusion under Flexible Tooling (RIFT): a review, Comp. Part A: Appl. Sci. Manuf. 27 (1996) 517-524.

[5] P. P. Parlevliet, H. E. Bersee, A. Beukers, Measurement of (post-)curing strain development with fibre Bragg gratings, Polym. Test. 29 (3) (2010) 291-301.

[6] T. Liu, G. Fernando, Processing of polymer composites: an optical fibre-based sensor system for on-line amine monitoring, Comp. Part A: Appl. Sci. Manuf. 32 (2001) 1561-1572.

[7] L. Merad, M. Cochez, S. Margueron, F. Jauchem, M. Ferriol, B. Benyoucef, P. Bourson, In-situ cure monitoring using optical fibre sensors - a comparative study, Polym. Test. 28 (2009) 42-45.

[8] G. Powell, P. Crosby, D. Waters, C. France, R. Spooncer, G. Fernando, In-situ cure monitoring using optical fibre sensors - a comparative study, Smart Mat. Struct. 7 (1998) $557-568$.

[9] S. Vacher, J. Molimard, H. Gagnaire, A. Vautrin, A Fresnel's reflection optical fiber sensor for thermoset polymer cure monitoring, Polym. Polym. Comp. 12 (4) (2004) 269-276. 
[10] V. Bhatia, D. Campbell, D. Sherr, T. D’Alberto, N. Zabaronick, G. Ten Eyck, K. Murphy, R. Claus, Temperature-insensitive and strain insensitive long-period grating sensors for smart structures, Opt. Eng. 36 (1997) 1872-1876.

[11] M. Xu, J.-L. Archambault, L. Reeckie, J. Dakin, Discrimination between strain and temperature effects using dual-wavelength fibre grating sensors, Elect. Let. 30 (13) (1994) $1085-1087$.

[12] Y. Han, S. Lee, C.-S. Kim, J. Kang, U.-C. Paek, Y. Chung, Simultaneous measurement of temperature and strain using long period fiber gratings with controlled temperature and strain sensitivities, Opt. Express 11 (5) (2003) 476-481.

[13] S. Triollet, L. Robert, E. Marin, Y. Ouerdane, Discriminated measures of strain and temperature in metallic specimen with embedded superimposed long and short fibre Bragg gratings, Meas. Sci. Tech. 22 (2011) 015202.

[14] H. J. Patrick, G. M. Williams, A. D. Kersey, J. R. Pedrazzani, A. M. Vengsarkar, Hybrid fiber Bragg grating/Long period fiber grating sensor for strain/temperature discrimination, IEEE Phot. Tech. Let. 8 (2006) 1223-1225.

[15] M. Song, S. Lee, S. Choi, B. Lee, Simultaneous Measurement of Temperature and Strain Using Two Fibre Bragg Gratings Embedded in a Glass Tube, Opt. Fibre Tech. 3 (2) (1997) 194-196.

[16] K. Kuang, L. Zhang, W. Cantwell, I. Bennion, Process monitoring of aluminium-foam sandwich structures based on thermoplastic fibre-metal laminates using fibre Bragg gratings, Comp. Sci. Tech. 65 (2005) 669-676.

[17] M. Mulle, F. Collombet, P. Olivier, R. Zitoune, C. Huchette, F. Laurin, Y.-H. Grunevald, Assessment of cure-residual strains through the thickness of carbon-epoxy laminates using FBGs Part II: Technological specimen, Comp. Part A: Appl. Sci. Manuf. 40 (2009) 1534-1544. 
[18] J. S. Leng, A. Asundi, Real-time cure monitoring of smart composite materials using extrinsic Fabry-Perot interferometer and fiber Bragg grating sensors, Smart Mat. Struct. 11 (2002) 249-255.

[19] V. Bhatia, Applications of long-period gratings to single and multi-parameter sensing, Opt. Express 4 (1999) 457-466.

[20] W. Jin, W. Michie, G. Thursby, M. Konstantaki, B. Culshaw, Simultaneous measurement of strain and temperature: error analysis, Opt. Eng. 36 (1997) 598-609.

[21] M. C. Kazilas, Acquisition and Interpretation of Dielectric Data For Thermoset Cure Monitoring, Ph.D. thesis, Cranfield University UK, 2003.

[22] Y. Wang, B. Han, D.W. Kim, A. Bar-Cohen, P. Joseph, Integrated Measurement Technique for Curing Process-Dependent Mechanical Properties of Polymeric Materials Using Fibre Bragg Grating, Exp. Mech. 48 (2008) 107-117.

[23] K. Magniez, A. Vijayan, N. Finn, Apparent Volumetric Shrinkage Study of RTM6 Resin During the Curing Process and Its Effect on the Residual Stresses in a Composite, Polym. Eng. Sci. 52 (2) (2012) 346-351.

[24] X. Aduriz, C. Lupi, N. Boyard, J.-L. Bailleul, D. Leduc, V. Sobotka, N. Lef"evre, X. Chapeleau, C. Boisrobert, D. Delaunay, Quantitative control of RTM6 epoxy resin polymerisation by optical index determination, Comp. Sci. Tech. 67 (2007) 3196-3201.

[25] J. Guemes, J. Menendez, Response of Bragg grating ber-optic sensors when embedded in composite laminates, Comp. Sci. Tech. 62 (2002) 959-966.

[26] F. Colpo, L. Humbert, P. Giaccari, J. Botsis, Characterization of residual strains in an epoxy block using an embedded FBG sensor and the OLCR technique, Comp. Part A: Appl. Sci. Manuf. 37 (4) (2006) 652-661.

\section{List of Figures}


Fig. 1. Thermocouple temperature (left), FBG and LPG wavelength shifts (right) vs. time recorded for the temperature cycle applied to the resin sample.

Fig. 2. Temperature (a) and strain (b) measured with the superimposed LPG/FBG sensor during the curing process.

Fig. 3. Strain versus temperature measured by the FBG/LPG sensor during the curing of the epoxy resin.

Fig. 4. Thermocouple temperature (left), LPG and FBG wavelength shifts (right) during the LRI process.

Fig. 5. Measurement of the resin ionic viscosity with DEA system during the first two phases (heating and isothermal plateau) of the LRI process.

Fig. 6. Temperature (a) and strain (b) measured with the superimposed LPG/FBG sensor during the LRI process. (c) and (d) are respectively the enlargement of (a) and (b).

Fig. 7. LPG spectra (a) and FBG spectra (b) at several times during the LRI process. 


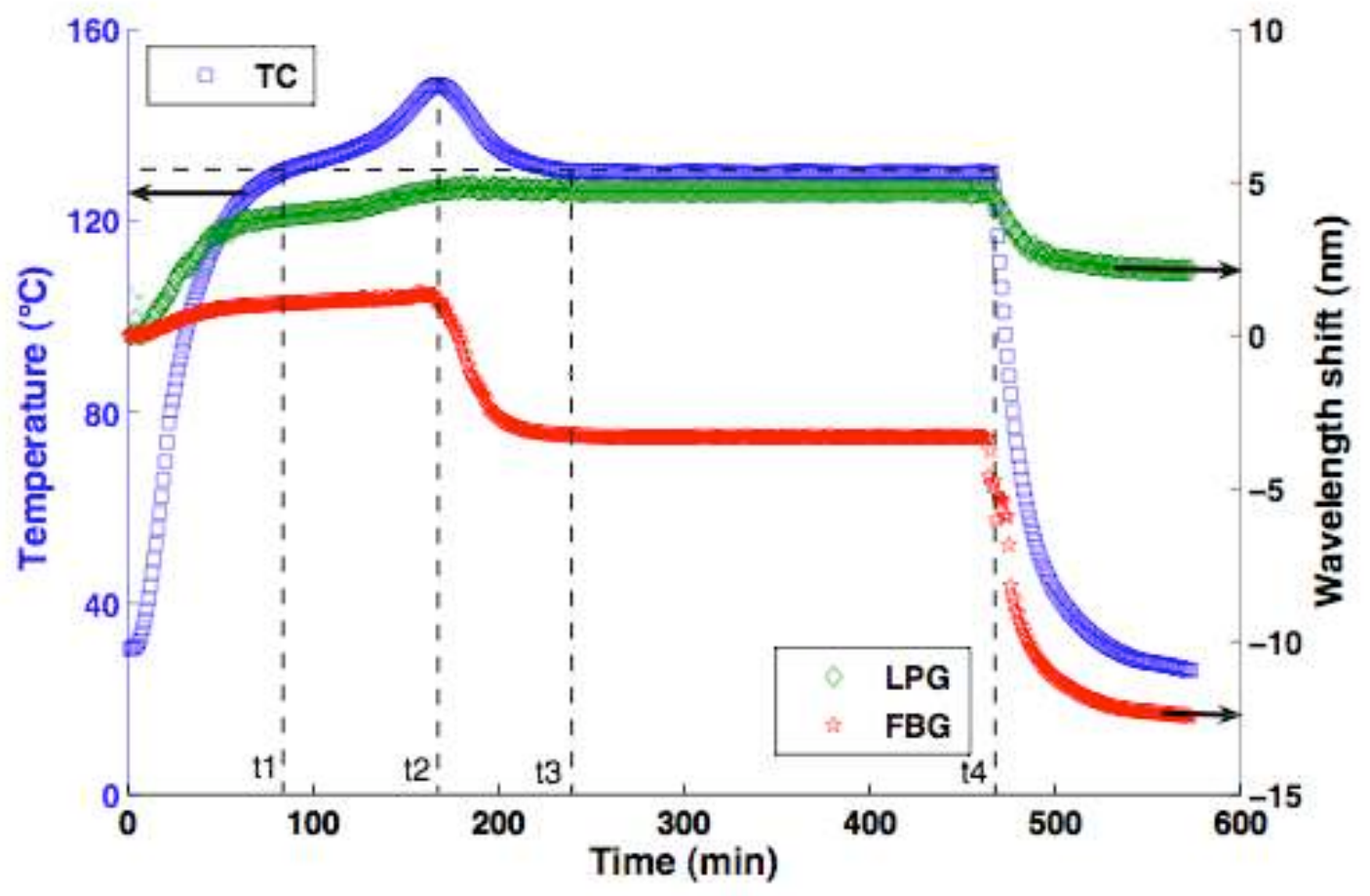

Fig. 1. Thermocouple temperature (left), FBG and LPG wavelength shifts (right) vs. time recorded for the temperature cycle applied to the resin sample. 
(a)

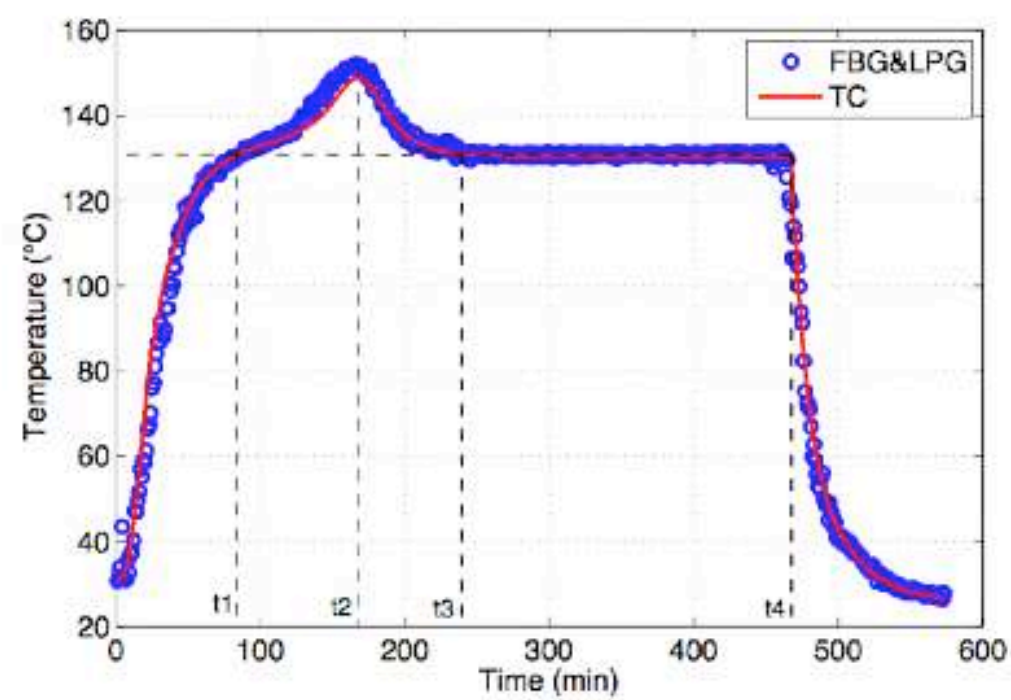

(b)

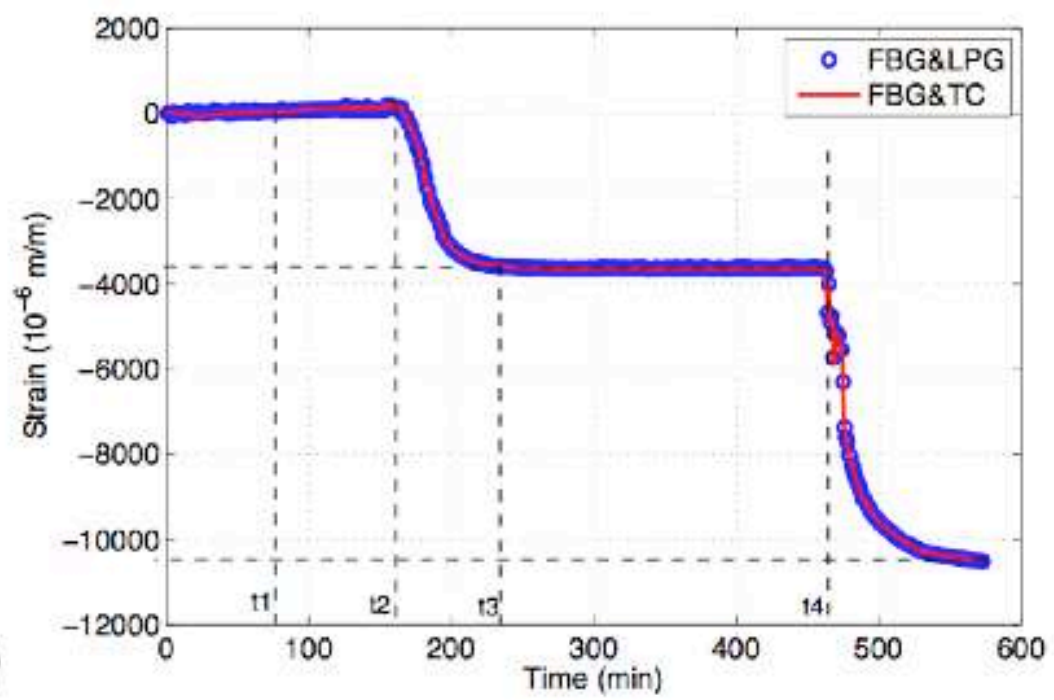

Fig. 2. Temperature (a) and strain (b) measured with the superimposed LPG/FBG sensor during the curing process. 


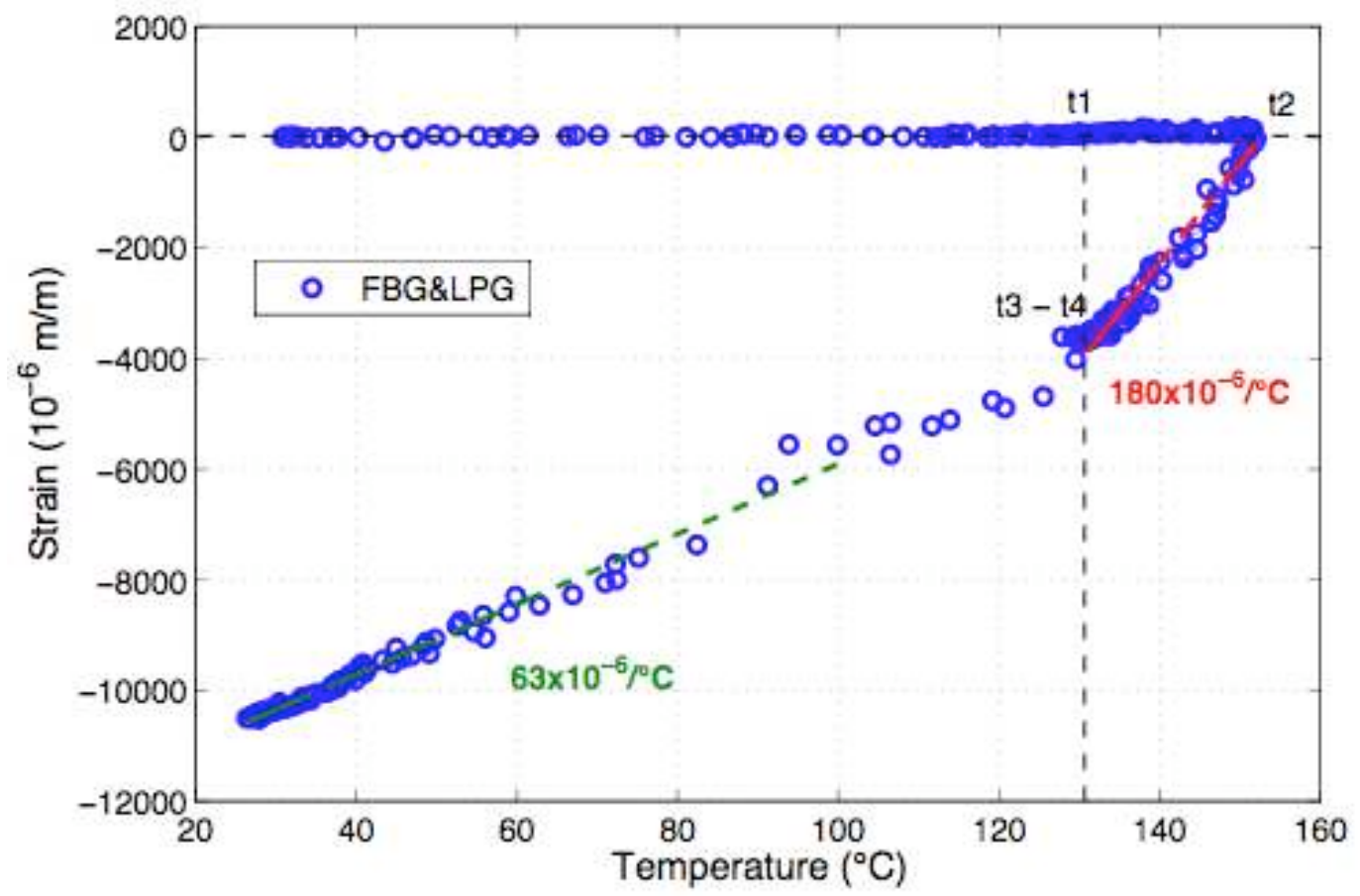

Fig. 3. Strain versus temperature measured by the FBG/LPG sensor during the curing of the epoxy resin. 


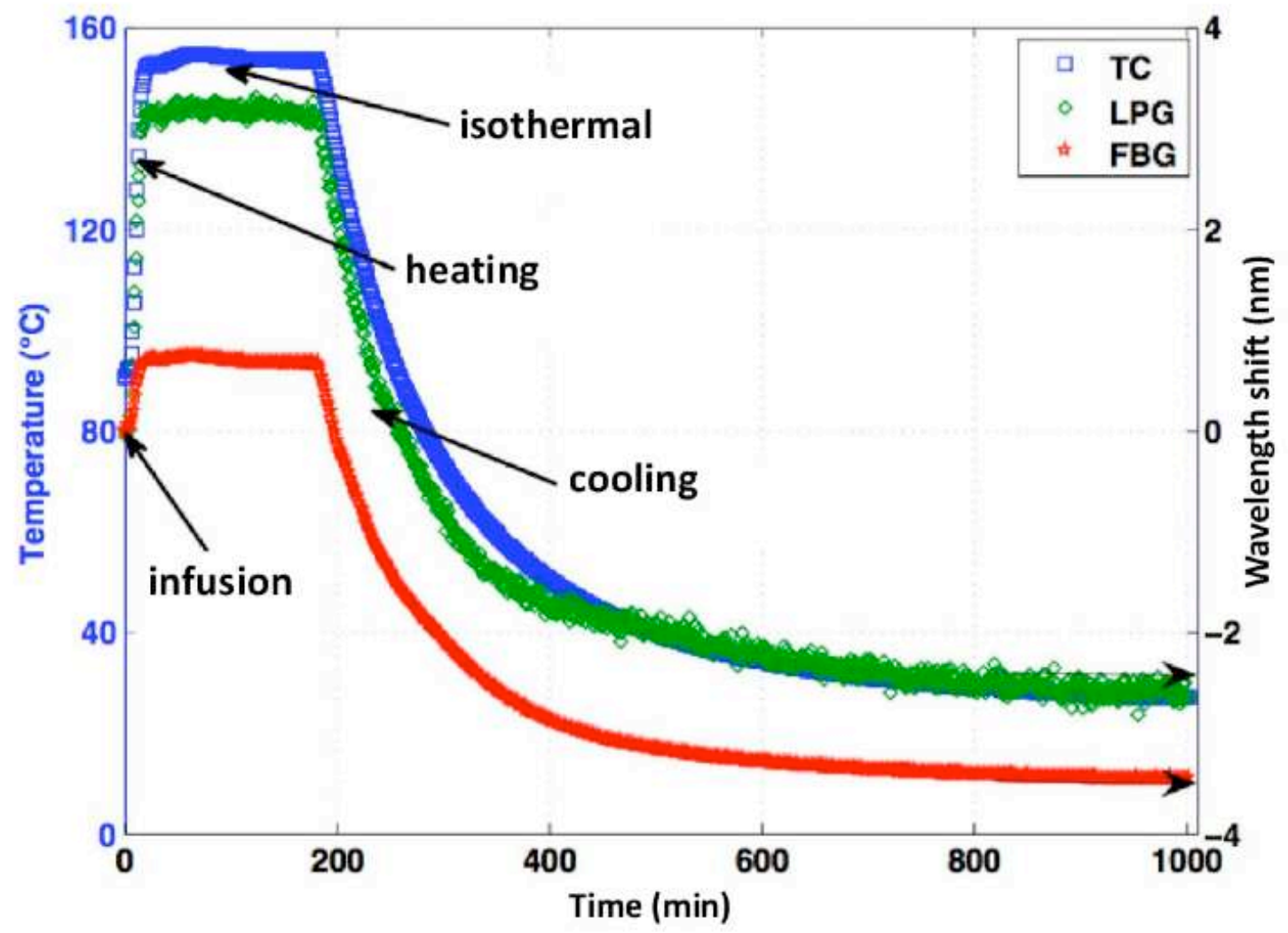

Fig. 4. Thermocouple temperature (left), LPG and FBG wavelength shifts (right) during the LRI process. 


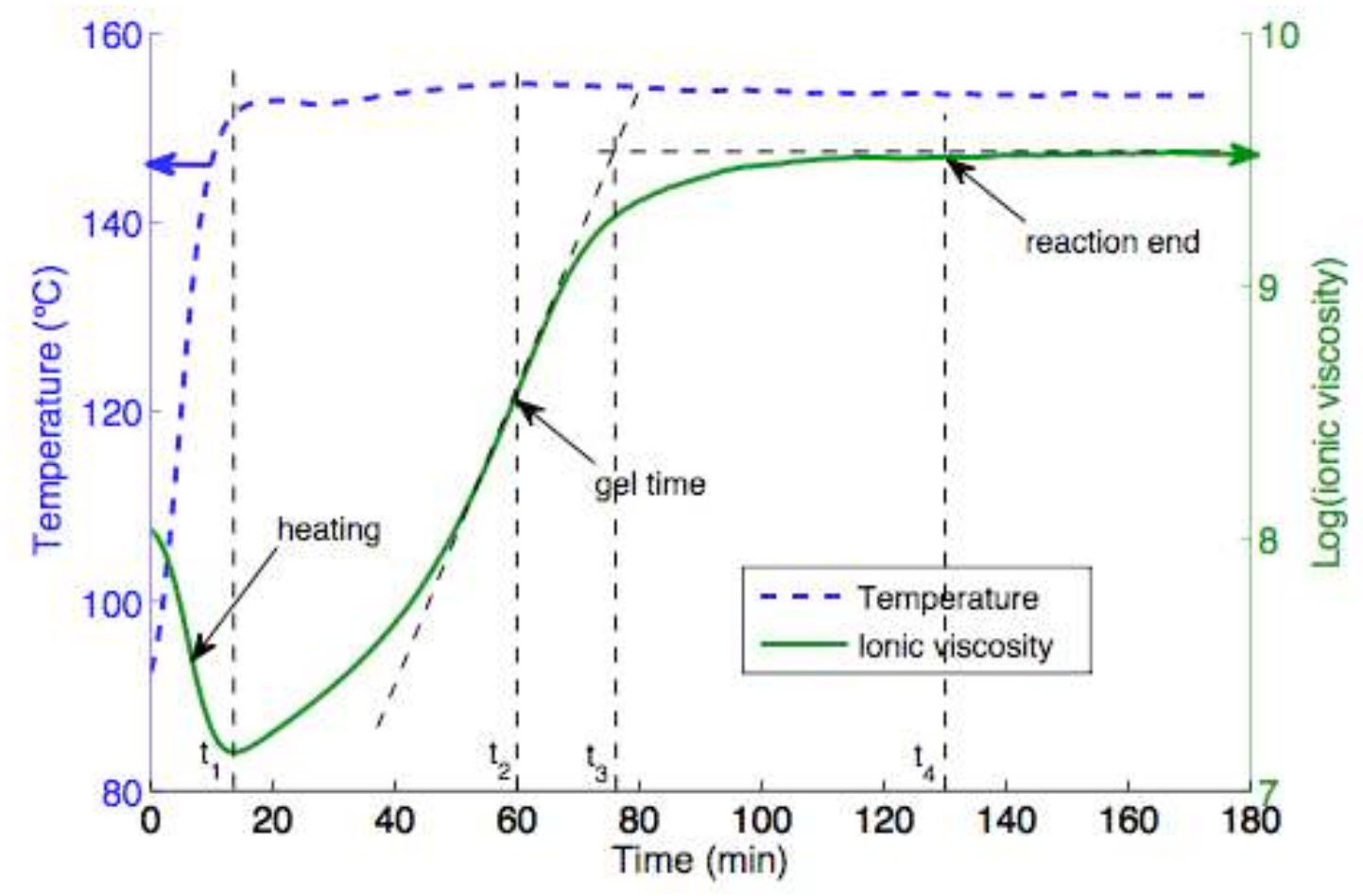

Fig. 5. Measurement of the resin ionic viscosity with DEA system during the first two phases (heating and isothermal plateau) of the LRI process. 

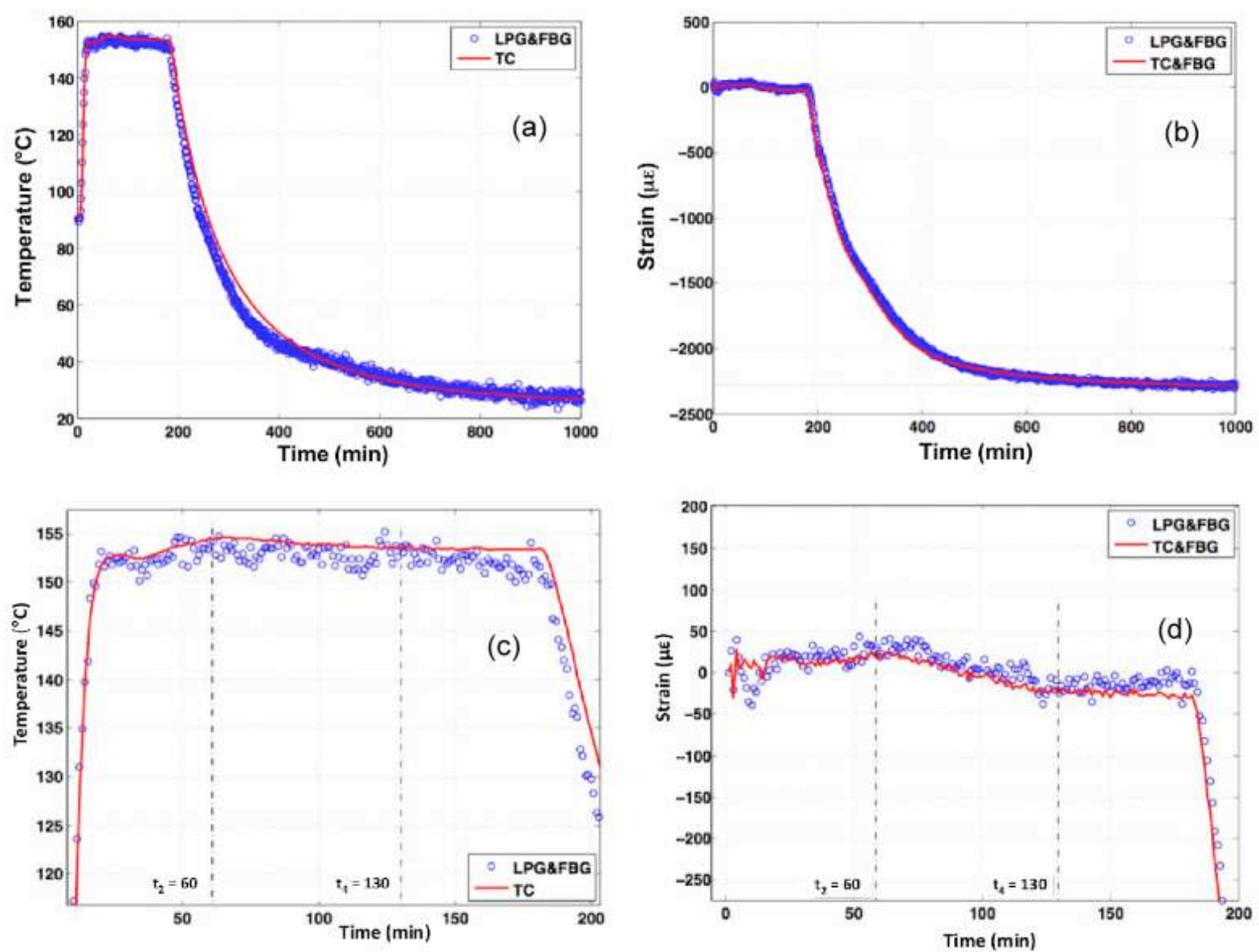

Fig. 6. Temperature (a) and strain (b) measured with the superimposed LPG/FBG sensor during the LRI process. (c) and (d) are respectively the enlargement of (a) and (b). 
(a)

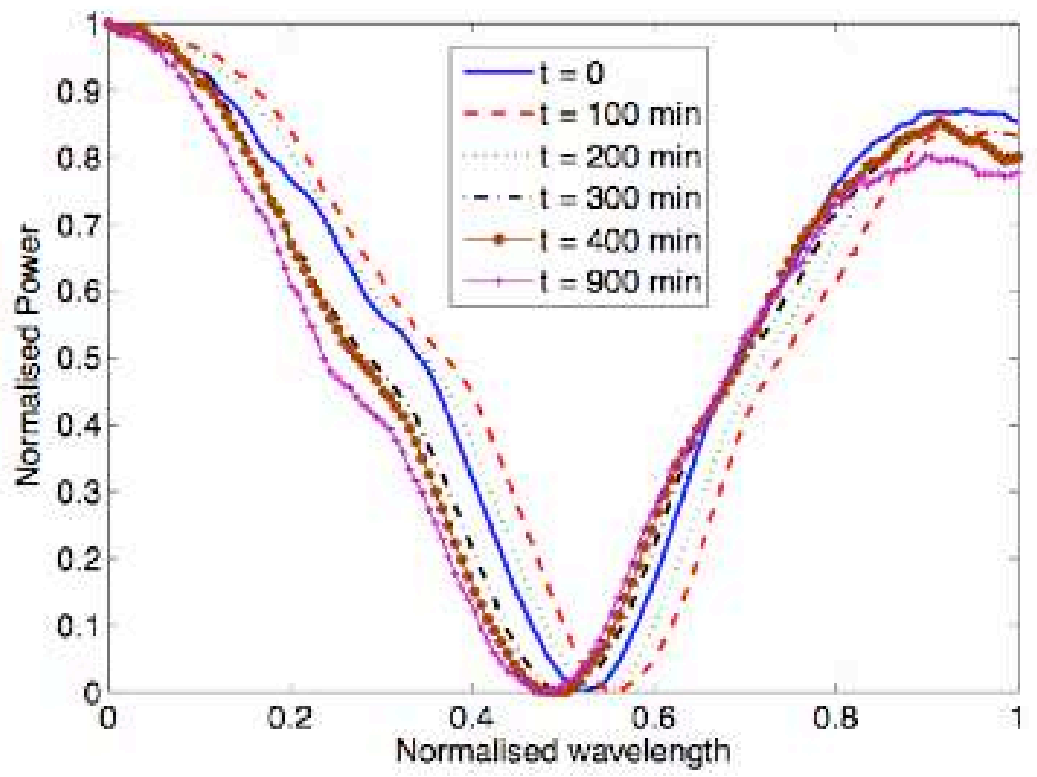

(b)

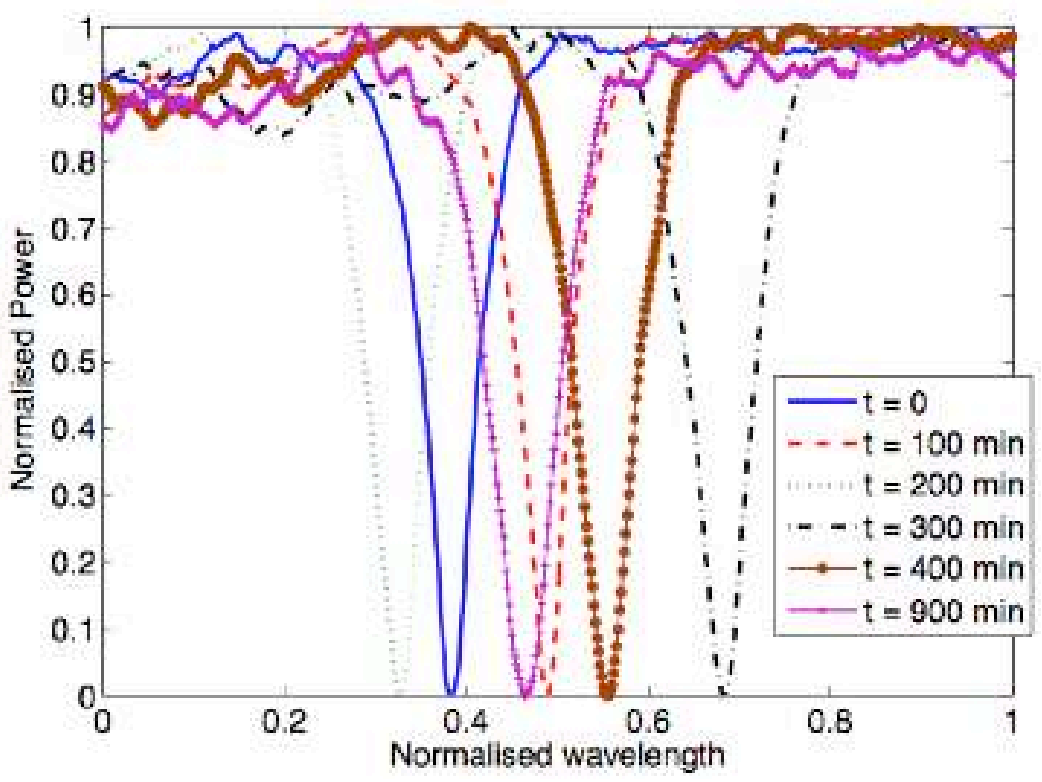

Fig. 7. LPG spectra (a) and FBG spectra (b) at several times during the LRI process. 